\title{
1 Modelling the electrical conductivity of soil in the Yangtze delta in three dimensions
}

3 H.Y. Li ${ }^{\mathrm{a}, *}$, B.P. Marchant ${ }^{\mathrm{b}}$, R. Webster ${ }^{\mathrm{c}}$

${ }_{4}{ }^{a}$ School of Tourism and Urban Management, Jiangxi University of Finance and

5 Economics, Nanchang 330013, China

${ }_{6}{ }^{\mathrm{b}}$ British Geological Survey, Keyworth, Nottingham NG12 5GG, UK

${ }_{7}{ }^{\mathrm{c}}$ Rothamsted Research, Harpenden AL5 2JQ, UK

8 Keywords:

9 Saline soil

10 Electrical conductivity

11 Three-dimensional variation

12 Space-time covariance functions

13

REML

E-BLUP

15

16

$17 *$ Corresponding author.

$18 \quad$ E-mail address: lihongyi1981@zju.edu.cn (H.Y. Li). 


\section{ABSTRACT}

Numerous processes, past and present, have given rise to lateral and vertical variation in the soil and to its individual properties such as its salinity and electrical conductivity. The resulting patterns of variation are complex and appear to comprise both random and deterministic components. The latter dominates vertically as trends in most soil profiles, and in the situation we describe it is prominent in the horizontal plane, too. Describing this variation requires flexible choice of covariance function. The processes of model estimation and prediction by kriging in three dimensions are similar to those in two dimensions. The extra complexity of the three-dimensional variation requires practitioners to appreciate fully the assumptions that their choices of model imply and to establish ways of testing the validity of these assumptions. We have examined several covariance functions more commonly used to describe simultaneously variation in space and time and adapted them to model three-dimensional variation in soil. We have applied these covariance functions to model the variation in salinity in reclaimed land in the Yangtze delta of China where the apparent electrical conductivity $\left(\mathrm{EC}_{\mathrm{a}}\right)$ has been measured at numerous points down to $1.1 \mathrm{~m}$. The models take into account random and deterministic components in both the horizontal and vertical dimensions. The most suitable mixed model was then used to krige the $\mathrm{EC}_{\mathrm{a}}$ on a fine grid from which three-dimensional diagrams of the salinity are displayed.

\section{Introduction}

It is now common practice to use geostatistical methods to model the horizontal variation of soil properties and to predict values at unvisited sites by some form of kriging (Webster and Oliver, 2007). In many instances one can treat the variation as the outcomes of intrinsically stationary correlated random processes and model the 
variation satisfactorily with one or other of the popular authorized variogram

functions. The random variation may be isotropic, so that one may disregard direction. Alternatively where the spatial correlation evidently varies with changes in direction one can often treat the anisotropy as geometric and elaborate the model in the form of a geometric anisotropic variogram function. Such a function permits the distance parameter(s) in the model to vary according to direction. If the variogram is bounded its sill is the same in all directions.

In three dimensions this assumption of a constant sill is much less likely to be appropriate for soil. The processes such as differential weathering, leaching and fluctuating ground water which lead to vertical variation differ substantially from the earth surface processes that act horizontally and on quite different spatial scales. This can lead to quite different horizontal and vertical sill variances, even after the removal of any trend components. More complex variograms or spatial covariance functions are required.

An analogous problem occurs when we model the variation of a property in both space and time, and several spatio-temporal correlation functions have been proposed (De Cesare et al., 2001; Kyriakidis et al., 1999).

In this paper we demonstrate that such functions can be used to represent the three-dimensional variation of a soil property, namely the soil's apparent electrical conductivity $\left(\mathrm{EC}_{\mathrm{a}}\right)$ which is commonly used as a proxy for soil salinity. We do so with sample data on $\mathrm{EC}_{\mathrm{a}}$ recorded in an ongoing investigation into the salinity in the Yangtze delta (Li et al., 2013; 2015).

\section{The setting}

The land in the coastal zone of Zhejiang Province south of China's Hangzhou Gulf of the Yangtze delta is formed of recent marine and fluvial deposits. Huge quantities of sediment are deposited in the delta each year, and as the delta builds so more of it can be empoldered and claimed for agriculture, in particular, for paddy rice. Rice will not grow well, if at all, in salty soil, however. Farmers, therefore, wish 
to be sure before they plant their rice that salt will not impair its growth. Farmers therefore wish to know that the soil is effectively free of salt before they attempt to grow the crop. They want accurate estimates of the soil's salinity, both laterally from place to place within their new fields and down the profile because the rice plants are susceptible to salt in the root zone from the surface to at least $1 \mathrm{~m}$. Ideally they would like three-dimensional maps of the salinity in their fields.

One can now monitor the soil's salinity using electromagnetic induction equipment such as the Geonics EM31 and EM38 instruments (McNeill, 1980). These devices measure the $\mathrm{EC}_{\mathrm{a}}$ of the soil, which is closely related to the soil's salinity. The EM38 is especially useful in that it can measure the $\mathrm{EC}_{\mathrm{a}}$ to approximately $1.5 \mathrm{~m}$ depth from the surface. One can use it therefore to obtain measures of the soil's salinity throughout the root zone of the rice without having to dig or bore into the soil to take samples.

In an earlier paper (Li et al., 2013) we described the Tikhonov regularization for converting the instrumental responses of the EM38 to $\mathrm{EC}_{\mathrm{a}}$ at ten depths in the soil in a 2.2-ha field that had been empoldered in 1996. We then modelled the three-dimensional variation in $\mathrm{EC}_{\mathrm{a}}$ as a series of correlated two-dimensional regionalized variables, one variable for each of the ten depths down to $1.1 \mathrm{~m}$, and kriged the $\mathrm{EC}_{\mathrm{a}}$ on a fine grid at those depths. We displayed the kriged predictions as a series of maps of EC, and built from the bottom upwards a three-dimensional block diagram. Since measurements from different depths were treated as different variables, discontinuities were evident in the predicted vertical profiles and $\mathrm{EC}_{\mathrm{a}}$ could not be predicted at depths where it was not measured.

The results revealed a trend in salinity across the field. In a second paper (Li et al., 2105), for which we had many more measurements in the topsoil, we were able to treat the data as the outcome of a linear mixed model (LMM) comprising both a fixed effect of the trend and a random residual from it and to estimate the parameters of the model by residual maximum likelihood (REML). Then by universal 
kriging we predicted the salinity at the nodes of a fine grid for mapping.

Figure 7 of the paper by Li et al. (2013) also showed what appeared to be a general increase in salinity with increasing depth. In an independent study in an adjacent field the authors found that in five of the nine profiles they measured there was indeed a steady increase in conductivity.

Our aim now is to model the full three-dimensional variation in salinity, taking into account both the lateral and vertical trends, and to use whatever models we fit to predict the salinity in the three dimensions by kriging.

\section{The data}

The field has an area of approximately 2.2 ha. The electrical conductivity of soil, recorded as $\mathrm{EC}_{\mathrm{a}}$, was measured with a Geonics EM38 conductivity meter at 56 nodes, approximately on a $20 \mathrm{~m} \times 20 \mathrm{~m}$ grid (Figure 1 ).

At each position, the readings were made using EM38 instruments with the coil configured both horizontally and vertically. The first $\mathrm{EC}_{\mathrm{a}}$ measurements were made on the ground surface to provide values of the soils $\mathrm{EC}_{\mathrm{a}}$ to theoretical depths of 0.75 and $1.5 \mathrm{~m}$, respectively. Then, the EM38 instrument was raised in increments of 0.1 $\mathrm{m}$ and readings were taken up to $0.6 \mathrm{~m}$. Further readings were taken at heights of $0.75,0.9,1.1,1.2$ and $1.5 \mathrm{~m}$ above the surface. The linear model described by Borchers et al. (1997) was applied to this set of measurements to estimate $\mathrm{EC}_{\mathrm{a}}$ at ten depths, namely $0.05,0.15,0.25,0.35,0.45,0.55,0.675,0.825,0.95$ and $1.05 \mathrm{~m}$, by second-order Tikhonov regularization. The diameters of the white circles in Figure 1 are proportional to the mean $\mathrm{EC}_{\mathrm{a}}$ across all ten depths. These values of $\mathrm{EC}_{\mathrm{a}}$ and their spatial coordinates comprise the data for our study. We use the following notation in referring them.

We denote by the vector $\mathbf{z}$ of length $n$ the full set of $n=560$ observations from $n_{\mathrm{s}}=56$ sites at $n_{\mathrm{d}}=10$ depths. We denote the spatial coordinates at which the observations were made by $\mathbf{x} \equiv\{x, y, d\}$ in which $x$ and $y$ are the two lateral dimensions and $d$ is depth. 
We draw attention here to two features of the data, displayed in Figure 2, and to the nature of the problem. Figure 2 shows (a) that there is a gradually increasing trend in $\mathrm{EC}_{\mathrm{a}}$ with increasing depth and (b) that the variance is not constant; the standard deviation is fairly constant down to $55 \mathrm{~cm}$, but increases thereafter down the profile. With these preliminary results in mind we nevertheless, proceed in stages, as follows.

\section{The general model}

We assume that the observed $\mathrm{EC}_{\mathrm{a}}$ can be represented by a linear mixed model (LMM):

$$
\mathbf{z}=\mathbf{M} \beta+\mathbf{u}
$$

As above, $\mathbf{z}$ denotes the vector of the $n=560$ observations. In addition $\mathbf{M}$ is the design matrix of the fixed effects; $\boldsymbol{\beta}$ is the parameter vector for those effects and $\mathbf{u}$ is the vector of random effects which are realizations of a multivariate Gaussian random process with mean zero and covariance matrix $\mathbf{C}$.

In the two-dimensional LMM of Li et al. (2015) for salinity in the top $10 \mathrm{~cm}$ of soil the best-fitting model had a quadratic spatial trend in the fixed effects (i.e. the columns of the $\mathbf{M}$ matrix were $1 \mathrm{~s}, x, y, x^{2}, y^{2}$ and $x y$, as displayed in Figure 1), and an isotropic two-dimensional spatial covariance function, $C(h)$, in which $h$ is a lag in horizontal distance only. Our aim here is to extend that model to describe quantitatively the variation in three dimensions. We might succeed by including depth, $d$, in the fixed effects or by estimating a covariance matrix that is a function of the three-dimensional lag vector separating the pairs of observations (i.e. $C=C(h, v)$ for vertical lag $v$ ), or a combination of the two. We itemize some of the possible extended models in the appendix below.

The parameters of our covariance functions could be estimated by the method-of-moments (Webster and Oliver, 2007). In this approach, point estimates of the expected squared differences between pairs of observations are calculated for 
several lags. Then the model parameters are selected such that there is a good match between the point estimates and the fitted covariance function. We previously used the method-of-moments to estimate our model which treated the $\mathrm{EC}_{\mathrm{a}}$ measurements from different depths as a series of correlated two-dimensional regionalized variables (Li et al., 2013). In our later paper, however, which looked specifically at two-dimensional variation ( $\mathrm{Li}$ et al., 2015), we found that better validation statistics resulted from models estimated by likelihood-based methods. This finding was not unexpected because the method-of-moments requires several subjective decisions. In particular, the practitioner must decide what lag bins to use and how to the allocate pairs of observations among them, and he or she must choose a suitable criterion to identify the best fitting model. Also, the method-of-moments does not account for the correlation between the different point estimates. In contrast, likelihood-based estimators estimate model parameters according to a statistical criterion that accounts fully for the correlations among the data.

Therefore, we estimate each model by maximum likelihood (ML) and compare the suitabilities of the models by calculating the Akaike Information Criterion (AIC):

$$
\mathrm{AIC}=2 k-2 \ln L,
$$

where $L$ is the likelihood and $k$ is the number of parameters in the model (Akaike, 1973). The preferred model is the one with the smallest AIC; we consider it the best compromise between quality of fit to the data and the model's complexity (number of parameters).

We have cross-validated the models by the leave-one-out method and calculated the standardized prediction errors:

$$
\theta_{i}=\frac{\left(z_{i}-\widehat{Z}_{i}\right)^{2}}{\sigma_{\mathrm{K}}^{2}(i)},
$$

where $z_{i}$ is the observation at site $i, \widehat{Z}_{i}$ is the kriged prediction at site $i$ when $z_{i}$ is excluded from the kriging predictor, and $\sigma_{\mathrm{K}}^{2}(i)$ is the corresponding kriging variance. If the errors are normally distributed then the $\theta_{i}$ will be a realization of a 
standardized chi-squared distribution with one degree of freedom. The mean of the $\theta_{i}$, say, $\bar{\theta}$, and usually reported as the mean squared deviation ratio (MSDR), then has expectation 1.0, and the median of $\theta_{i}, \tilde{\theta}$ or medSDR, has the expected value 0.455 for a standard chi-squared distribution.

We follow Li et al. (2015) and assume a quadratic horizontal spatial trend in the fixed effects. We add a linear trend with $d$ which reflects the observed relationship between $\mathrm{EC}_{\mathrm{a}}$ and $d$ (Fig. 2). We compare various covariance functions. In the discussion below we denote authorized covariance functions of (i) horizontal lag, (ii) vertical lag and (iii) horizontal and vertical lag by $C_{\mathrm{H}}, C_{\mathrm{V}}$ and $C_{\mathrm{HV}}$ respectively.

Our initial covariance model is a second-order stationary Matérn function (Matérn, 1960; Marchant and Lark, 2007):

$$
\begin{aligned}
& C(h, v)=c_{1}\left\{\frac{1}{2^{\nu-1}} \Gamma(\nu)\left(\frac{\sqrt{h^{2}+v^{2}}}{a}\right)^{\nu} K_{\nu}\left(\frac{\sqrt{h^{2}+v^{2}}}{a}\right)\right\} \text { for } \sqrt{h^{2}+v^{2}}>0 \\
& C(h, v)=c_{0} \text { for } \sqrt{h^{2}+v^{2}}=0
\end{aligned}
$$

where $c_{0}$ is the nugget variance, $c_{1}$ is the sill variance of the correlated structure, $a$ is a spatial parameter, $\nu$ is a smoothness parameter, $K_{\nu}$ is a modified Bessel function of the second kind of order $\nu$ (Abramowitz and Stegun, 1972) and $\Gamma$ is the gamma function.

Though this isotropic model is our starting point, we recognize that it is highly unlikely to be optimal, for that would imply identical covariance functions for the horizontal and vertical dimensions. The variation is almost certain to be anisotropic. Anisotropy is commonly accommodated in covariance functions via an affine transformation:

$$
C(h, v)=C_{\mathrm{HV}}\left(\sqrt{h^{2}+\alpha v^{2}}\right) .
$$

Here, $h$ and $v$ are the lags in the horizontal and vertical dimensions, which are denoted by the subscripts $\mathrm{H}$ and $\mathrm{V}$. The parameter $\alpha$ stretches or contracts the vertical range of spatial correlation relative to the horizontal range. The model still requires us to assume that the sills are identical in the horizontal and vertical dimensions, however. 
More flexible three-dimensional covariance functions have been devised to represent the spatial and temporal variation of properties. These functions are reviewed by De Cesare et al. (2001) and Kyriakidis and Journel (1999). The simplest space-time models are said to be separable. The spatial correlation is independent of the temporal correlation. Separable functions can be formed from the sum or product of a spatial and a temporal covariance function. Rouhani and Myers (1990) pointed out that the sum sometimes leads to singular kriging equations, and the assumption of independent spatial and temporal correlation functions is rather limiting.

Therefore several non-separable models have been proposed. Two of the most widely used (written in terms of horizontal and vertical rather than spatial and temporal lags) are the sum metric model:

$$
C(h, v)=C_{\mathrm{H}}(h)+C_{\mathrm{V}}(v)+C_{\mathrm{HV}}\left(\sqrt{h^{2}+\alpha v^{2}}\right) .
$$

and the product sum model:

$$
C(h, v)=C_{\mathrm{H}}(h)+C_{\mathrm{V}}(v)+k C_{\mathrm{H}}(h) C_{\mathrm{V}}(v),
$$

where $k>0$ is a parameter. Both of these models permit different sills and distance parameters in the horizontal and vertical dimensions, and they account for the dependence between the spatial correlations in each dimension.

All of the models described so far require the assumption that the random effects are stationary. This means that the covariances are functions of the lags between pairs of points and only of the lags; they do not depend on the specific locations of the points. A further complication in our study is that not only is there a trend of increasing $\mathrm{EC}_{\mathrm{a}}$ down the profile but also an increase in the variance - see Fig. 2. This increasing variance can be accommodated if the covariance matrix is scaled on both sides by a diagonal matrix $\mathbf{S}$. Thus the covariance matrix becomes SCS where the elements of the main diagonal of $\mathbf{S}$ are a function of location. We refer to this function as a scaling function, $S(d)$. Our chosen scaling functions are linear, quadratic and cubic polynomials of $\ln (d)$ and a discontinuous function where a 
different scaling value is estimated for each depth. We used polynomials of $\ln (d)$

rather than polynomials of $d$ because $\ln (d)$ had a stronger linear correlation with the standard deviation. We thus have LMMs comprising random and fixed effects.

The AIC, Equation (2), is based on maximum likelihood (ML) estimates of the parameters. There is a small bias, however, in ML estimates of variance parameters in the presence of fixed effects. So, once we have determined the most suitable model for the LMM we re-estimate the parameters by REML. Then we use the empirical best linear unbiased predictor (E-BLUP) or universal kriging predictor (Lark et al., 2006) to predict the $\mathrm{EC}_{\mathrm{a}}$ on a regular three-dimensional grid. The REML estimator minimizes the bias, but the residual likelihood cannot be used to calculate the AIC. Then we use the empirical best linear unbiased predictor (E-BLUP) or universal kriging predictor (Lark et al., 2006) to predict the $\mathrm{EC}_{\mathrm{a}}$ on a regular three-dimensional grid. There is a small bias in ML estimates of variance parameters in the presence of fixed effects. The REML estimator minimizes this bias, but the residual likelihood cannot be used to calculate the AIC, Equation (2).

\section{Results}

The summary validation statistics for the model with stationary isotropic random effects might be considered acceptable (Table 1). The mean square deviation ratio, MSDR, is 1.00 , and the medSDR is 0.29 .

Including geometric anisotropy in the models, however, diminishes the AIC substantially. There is a further decrease in the negative log-likelihood when the sum metric covariance function is used. The additional parameters in this model cause the AIC to increase, however. For models with stationary random effects the smallest AIC is obtained when the covariance function is a product sum model. The ML estimated variogram for this model appears to be consistent with the method-of-moments point estimates in all dimensions (Fig. 3). These point estimates, however, do not vary with depth. When the horizontal variograms for the separate depths are plotted individually the ML model appears to over-estimate the variogram 
near the surface of the soil and to under-estimate it at greater depths. We also see that the MSDR is considerably less than 1 near the surface and considerably greater than 1 for great depths (Fig. 5).

We could overcome some of these shortcomings by using non-stationary covariance matrices - see models 5-8 in the appendix. This adaptation led to further decreases in the AIC. The smallest AIC was achieved for the model with a unique scaling value for each depth, and the cubic polynomial led to the smallest AIC for a continuous scaling function. In Figs 4 and 5 we see that the cubic (red) and discontinuous (green) scaling functions lead to better fitted horizontal variograms across the several depths and that the MSDR for the different depths do not deviate so far from 1.0.

We favour the non-stationary model with a cubic scaling function since this can be used to predict $\mathrm{EC}_{\mathrm{a}}$ and hence soil salinity at any depth, whereas the model with discontinuous scaling function is limited to the depths at which soil salinity was measured. Figure 6 shows the kriged predictions from this model at several different depths. The quadratic horizontal trend and linearly increasing trend in salinity with depth are clearly evident.

\section{Discussion}

In many respects the procedures for estimating geostatistical models in three-dimensions are the same as those in two-dimensions. The observed measurements can be treated as a realization of an LMM. These models can be estimated by ML and the suitability of different fixed and random effects structures in the model can be compared via the AIC. Also, one can validate these models by calculating the MSDR.

The primary difference in the three-dimensional case is the potential for more complex patterns of variation and hence the existence of more ways in which the observed data can deviate from the assumed model. When we decide on the structure of the LMM we need to look for trends in expected values and variances both 
horizontally and vertically. We have seen that calculation of the MSDR averaged over the entire set of data is insufficient to validate these models. This summary statistic can disguise large deviations from the assumed model. Instead it is important that we understand the assumptions that our models imply and devise tests of the appropriateness of these assumptions. For example, we tested the assumption that the random effects were independent of depth by looking individually at the MSDR for each depth and we established that this assumption should be relaxed.

We could identify the best fitting model from our list of candidate models. However, the fit was by no means perfect. The medSPE was rather less than 0.45 and there were still some depths where the MSDR deviated from 1 . This indicates that further generalizations of the geostatistical model might be required.

In Fig 6, the quadratic horizontal trend and linearly increasing trend in salinity with depth are clearly evident in the field studied. The predictions vary smoothly in both the horizontal and vertical directions. This contrasts with the corresponding graphs in Li et al. (2013) where there were discontinuities in the predictions down the profile. Those discontinuities resulted from measurements from the different soil depths being treated as different variables.

However, the true value in our statistical model is that we have increased confidence that the uncertainty of our predictions has been reliably quantified. Therefore farmers can account for this uncertainty when they decide whether or not to grow rice. For example, rather than considering the expected $\mathrm{EC}_{\mathrm{a}}$ it might be relevant to explore the risk or probability that the soil salinity exceeds a critical threshold at each location. The FAO (1976) suggests that soil salinity equivalent to an $\mathrm{EC}_{\mathrm{a}}$ of $123 \mathrm{mS} \mathrm{m}{ }^{-1}$ is likely to lead to a $25 \%$ reduction in rice yield compared with non-saline soil. Since the kriging predictor yields both a prediction of $\mathrm{EC}_{\mathrm{a}}$ and an estimate of the prediction interval at each point in the field we can easily determine the probability that this threshold is exceeded (Fig 7). Thus we see that in the majority of the field and particularly at depth it is very likely that salinity will 
lead to loss of yield.

\section{Acknowledgments}

This research was supported by a grant from the National High Technology Research and Development Program of China (No 2013AA102301), the National Science Foundation of China (No 41101197;41561049), Ministry of Education, Humanities and Social Science Prject (No 10YJC910002) and Natural Science Foundation of Jiangxi Province (No 20114AB2 13017). We thank the bodies mentioned above and Professor Z. Shi for their support and Rothamsted Research for its hospitality. Our paper is published with the permission of the director of the British Geological Survey.

\section{References}

Abramowitz, M., Stegun, I.A., 1972. Handbook of Mathematical Functions with Formulas, Graphs, and Mathematical Tables. Wiley-Interscience, New York.

Akaike, H., 1973. Information theory and an extension of the maximum likelihood principle. In: Second International Symposium on Information Theory (eds B.N. Petrov and F. Csáki), pp 267-281. Akadémiai Kiadó, Budapest.

Borchers, B., Uram, T., Hendrickx, J.M.H., 1997. Tikhonov regularization of electrical conductivity depth profiles in field soils. Soil Science Society of America Journal 61, 1004-1009.

De Cesare, L., Myers, D.E., Posa, D., 2001. Estimating and modelling space-time correlation structures. Statistics \& Probability Letters 51, 9-14.

FAO, 1976. Prognosis of salinity and alkalinity. Soils Bulletin No 31, FAO, Rome.

Kyriakidis, P.K., Journel, A.G., 1999. Geostatistical space-time models: A review. Mathematical Geology 31, 651-684. 
Lark, R.M., Cullis, B.R., Welham, S.J., 2006. On spatial prediction of soil properties in the presence of a spatial trend: the empirical best linear unbiased predictor (E-BLUP) with REML. European Journal of Soil Science 57, 787-799.

Li, H.Y., Shi, Z., Webster, R., Triantifilis, J., 2013. Mapping the three-dimensional variation of soil salinity in a rice-paddy soil. Geoderma 195-196, 31-41.

Li, H.Y., Webster, R., Shi, Z., 2015. Mapping soil salinity in the Yangtze delta: REML and universal kriging (E-BLUP) revisited. Geoderma 237-238, 71-77.

Marchant, B.P., Lark, R.M. 2007. The Matérn variogram model: Implications for uncertainty propagation and sampling in geostatistical surveys. Geoderma 140, $337-345$.

Matérn, B., 1960. Meddelanden från Statens Skogsforskningsinstitut 49, 1-144.

McNeill, J.D., 1980. Electromagnetic Terrain Conductivity Measurement at Low Induction Numbers. Techical Note TN-6. Geonics Limited, Mississauga, Ontario.

Rouhani, S., Myers, D.E., 1990. Problems in space-time kriging of geohydrological data. Mathematical Geology 22, 611-623.

Webster, R., Oliver, M.A., 2007. Geostatistics for Environmental Scientists, 2nd Edition. John Wiley and Sons, Chichester. 


\section{Appendix}

Below, we list the parametric functions for the specific forms of the LMM,

Equation (1), considered in the paper. For all of these models the columns of the

design matrix for the fixed effects, $\mathbf{M}$, are $1 \mathrm{~s}, x, y, d, x^{2}, y^{2}$ and $x y$. All covariance functions are Matérn functions, Equation (4).

Model 1, isotropic

$S(d)=\alpha_{0}$, $C(h, v)=C_{\mathrm{HV}}\left(\sqrt{h^{2}+v^{2}}\right)$.

Model 2, geometric anisotropic

$$
S(d)=\alpha_{0},
$$$$
C(h, v)=C_{\mathrm{HV}}\left(\sqrt{h^{2}+\alpha v^{2}}\right) .
$$

Model 3, Sum metric

$$
S(d)=\alpha_{0},
$$

$$
C(h, v)=C_{\mathrm{H}}(h)+C_{\mathrm{V}}(v)+C_{\mathrm{HV}}\left(\sqrt{h^{2}+\alpha v^{2}}\right) .
$$

\section{Model 4, Product sum}

$$
S(d)=\alpha_{0},
$$

$$
C(h, v)=C_{\mathrm{H}}(h)+C_{\mathrm{V}}(v)+k C_{\mathrm{H}}(h) C_{\mathrm{V}}(v) .
$$

\section{Model 5, Product sum}

$$
\begin{aligned}
& S(d)=\alpha_{0}+\alpha_{1} \ln (d), \\
& C(h, v)=C_{\mathrm{H}}(h)+C_{\mathrm{V}}(v)+k C_{\mathrm{H}}(h) C_{\mathrm{V}}(v) .
\end{aligned}
$$

Model 6, Product sum

$$
\begin{aligned}
& S(d)=\alpha_{0}+\alpha_{1} \ln (d)+\alpha_{2}\{\ln (d)\}^{2}, \\
& C(h, v)=C_{\mathrm{H}}(h)+C_{\mathrm{V}}(v)+k C_{\mathrm{H}}(h) C_{\mathrm{V}}(v) .
\end{aligned}
$$

Model 7, Product sum 
$\begin{array}{ll}381 & S(d)=\alpha_{0}+\alpha_{1} \ln (d)+\alpha_{2}\{\ln (d)\}^{2}+\alpha_{3}\{\ln (d)\}^{3}, \\ { }_{382} & C(h, v)=C_{\mathrm{H}}(h)+C_{\mathrm{V}}(v)+k C_{\mathrm{H}}(h) C_{\mathrm{V}}(v) .\end{array}$

383

384

385

Model 8, Product sum

$S(d)=\alpha_{i}$ if $d=d_{i}$,

$C(h, v)=C_{\mathrm{H}}(h)+C_{\mathrm{V}}(v)+k C_{\mathrm{H}}(h) C_{\mathrm{V}}(v)$.

The $d_{i}$ for $i=1,2, \ldots, 10$ are the depths at which $\mathrm{EC}_{\mathrm{a}}$ was observed, and the $\alpha_{i}$ are parameters. 
Table 1

Validation statistics of the fitted three-dimensional models.

\begin{tabular}{lllllll} 
Model & $C(h, v)$ function & Scaling function & $\ln L$ & AIC & MSDR & medSDR \\
\hline 1 & Isotropic & Constant & 325.58 & -619.2 & 1.00 & 0.29 \\
2 & Geometric anisotropic & Constant & 549.47 & -1073.0 & 1.05 & 0.15 \\
3 & Sum metric & Constant & 553.01 & -1066.0 & 1.00 & 0.15 \\
4 & Product sum & Constant & 566.57 & -1097.2 & 1.08 & 0.17 \\
5 & Product sum & Linear & 648.15 & -1258.3 & 1.05 & 0.24 \\
6 & Product sum & Quadratic & 648.18 & -1256.4 & 1.05 & 0.24 \\
7 & Product sum & Cubic & 654.67 & -1267.4 & 1.05 & 0.23 \\
8 & Product sum & Discontinuous & 670.48 & -1285.0 & 1.03 & 0.26 \\
\hline \hline
\end{tabular}




\section{Figure captions}

Fig. 1 Positions of the 56 sampling locations shown by the white circles, the diameters of which are proportional to the mean $\mathrm{EC}_{\mathrm{a}}$ across all ten depths at those points. The grey layers show the horizontal quadratic trend surface fitted to those values.

Fig. 2 (a) Mean $\mathrm{EC}_{\mathrm{a}}$ plotted against depth; (b) standard deviation of $\mathrm{EC}_{\mathrm{a}}$ plotted against depth.

Fig. 3 Method-of-moments point estimates (black discs) and ML estimated stationary product sum model (curves) of the variogram plotted in the (a) horizontal, (b) vertical, (c) horizontal and displaced by $0.1 \mathrm{~m}$ vertically, (d) vertical and displaced by $20 \mathrm{~m}$ horizontally dimensions.

Fig. 4. (a)-(j) Horizontal method of moments point estimates (discs) and ML estimated product sum models (curves) for depths (a) 0.05, (b) 0.15, (c) 0.25, (d) 0.35, (e) 0.45, (f) 0.55, (g) 0.68, (h) 0.83, (i) 0.95 and (j) $1.05 \mathrm{~m}$. Scaling functions are constant (black), cubic (red) and discontinuous (green).

Fig. 5. MSDR (mean square deviation ratio) against depth for the ML estimated product sum models with constant (black), cubic (red) and discontinuous (green) scaling functions.

Fig. 6. Three-dimensional kriged predictions of $\mathrm{EC}_{\mathrm{a}}$ in $\mathrm{mS} \mathrm{m}^{-1}$.

Fig. 7. The probability that $\mathrm{EC}_{\mathrm{a}}$ exceeds $123 \mathrm{mS} \mathrm{m}^{-1}$. 


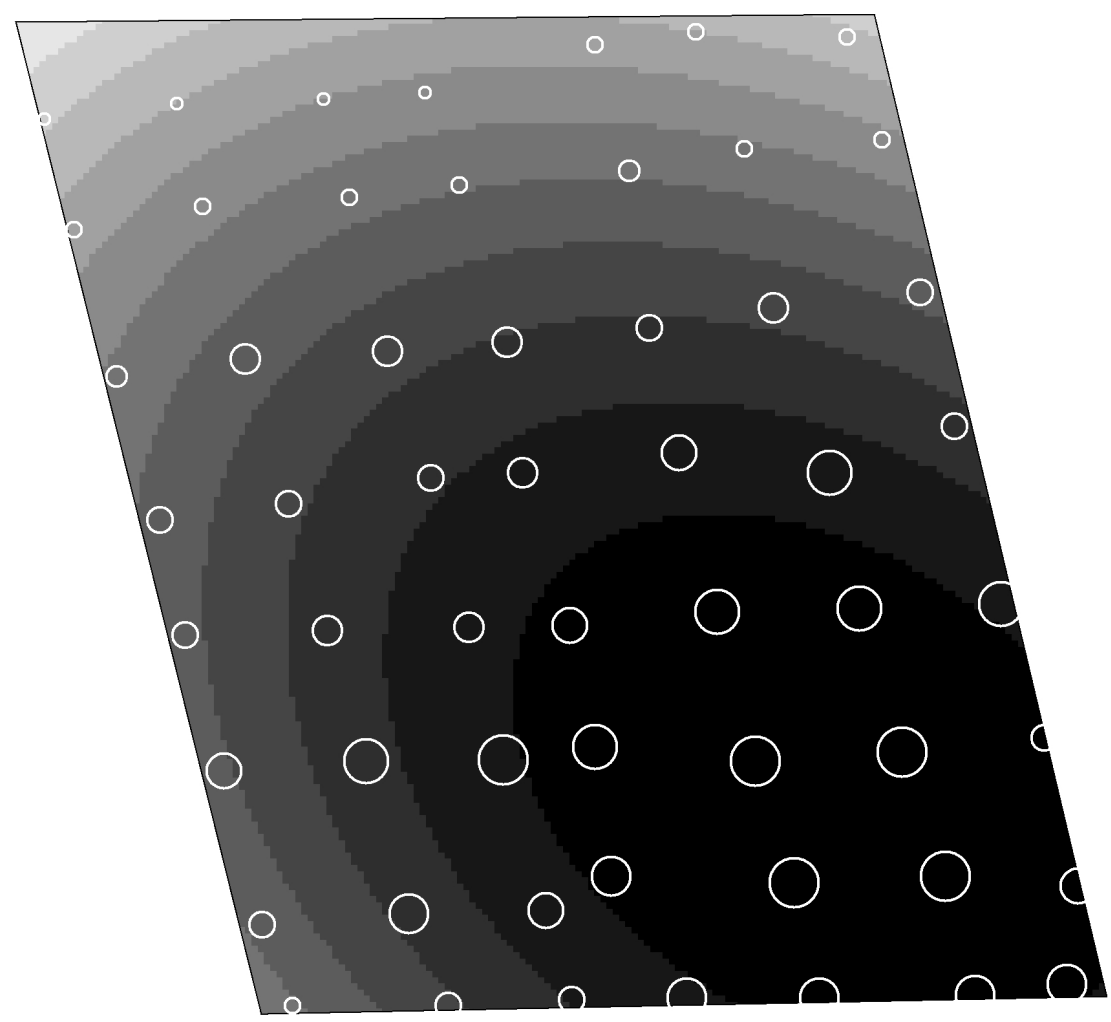

Fig. 1 Positions of the 56 sampling locations shown by the white circles, the diameters of which are proportional to the mean $\mathrm{EC}_{\mathrm{a}}$ across all ten depths at those points. The grey layers show the horizontal quadratic trend surface fitted to those values. 

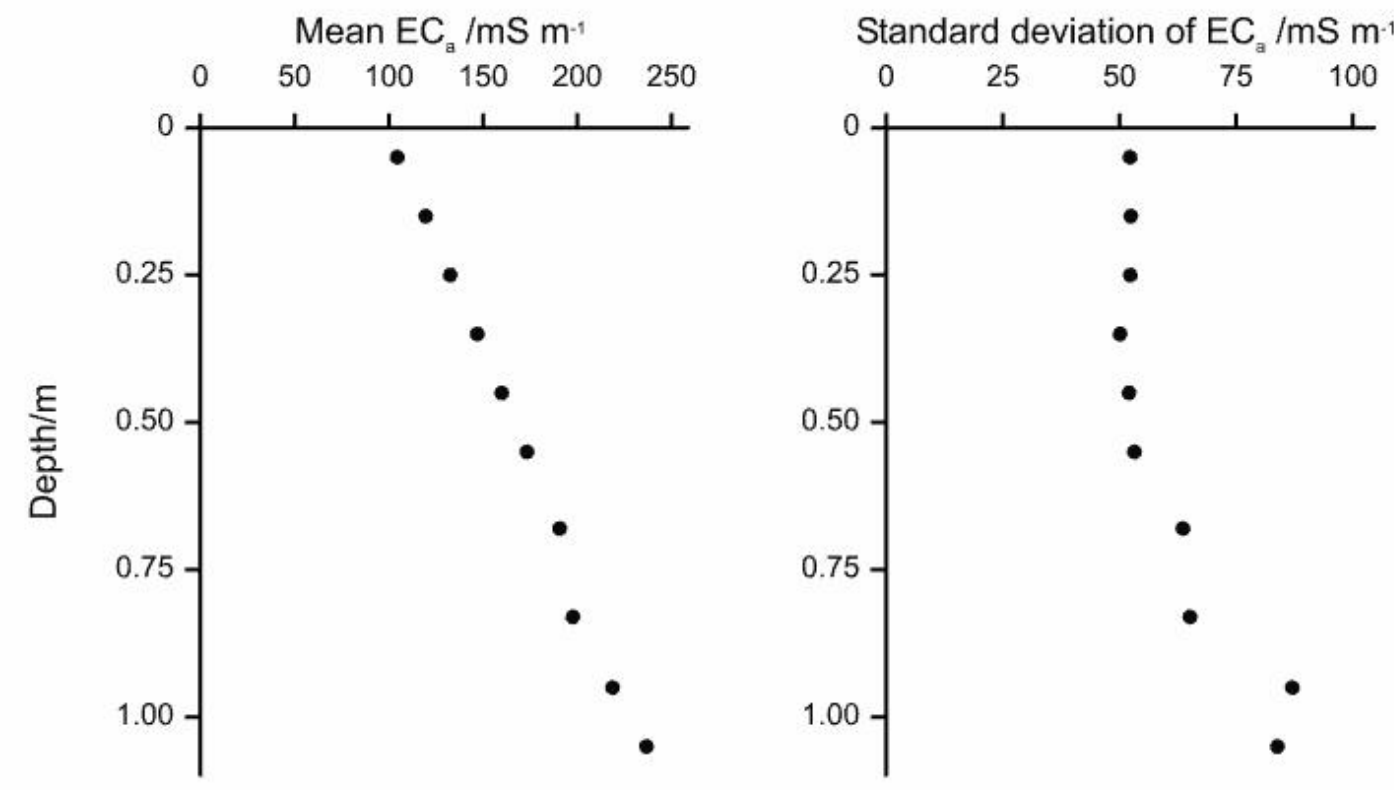

Fig. 2 (a) Mean $\mathrm{EC}_{\mathrm{a}}$ plotted against depth; (b) standard deviation of $\mathrm{EC}_{\mathrm{a}}$ plotted against depth. 

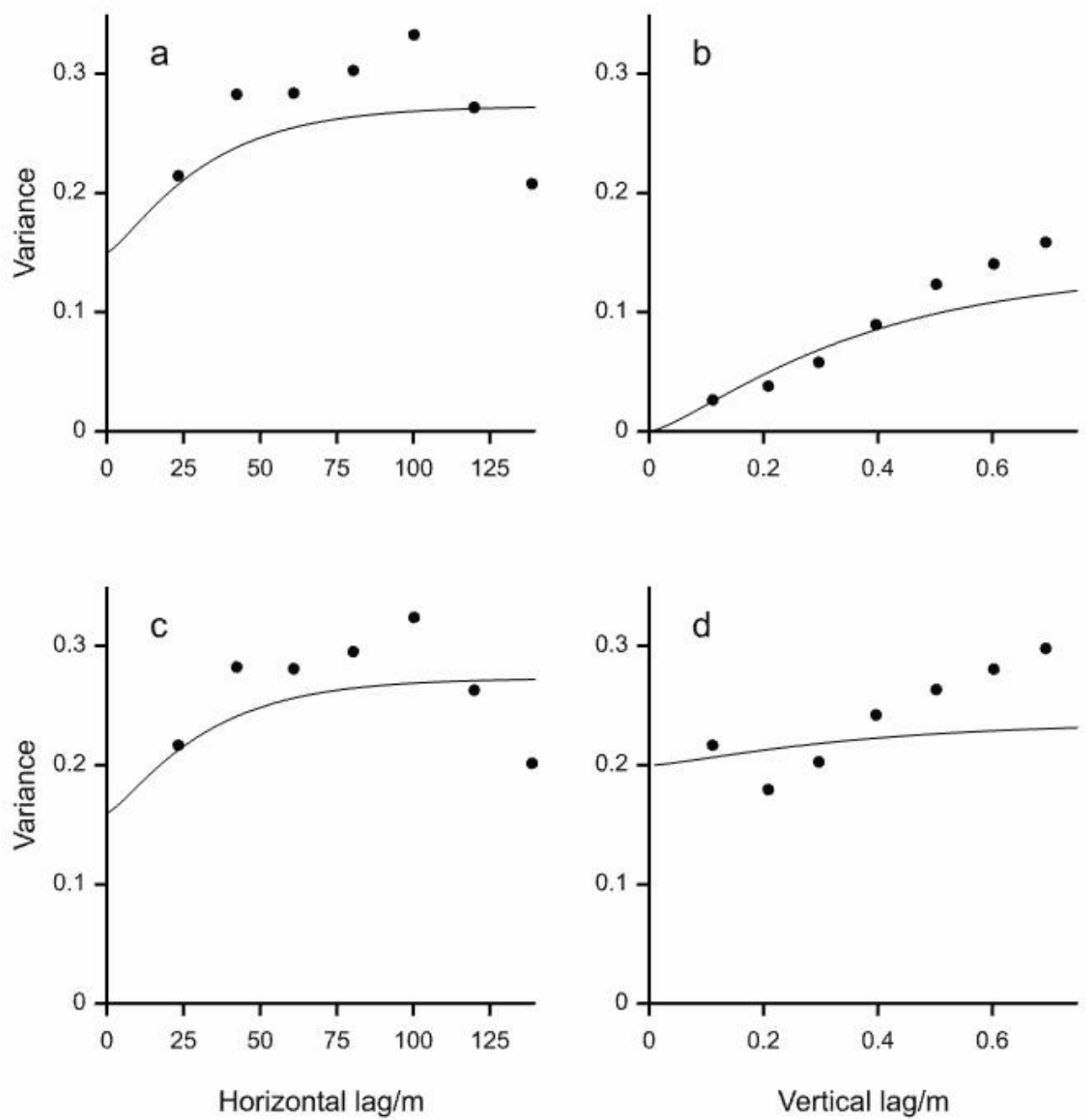

Fig. 3 Method-of-moments point estimates (black discs) and ML estimated stationary product sum model (curves) of the variogram plotted in the (a) horizontal, (b) vertical, (c) horizontal and displaced by $0.1 \mathrm{~m}$ vertically, (d) vertical and displaced by $20 \mathrm{~m}$ horizontally dimensions. 

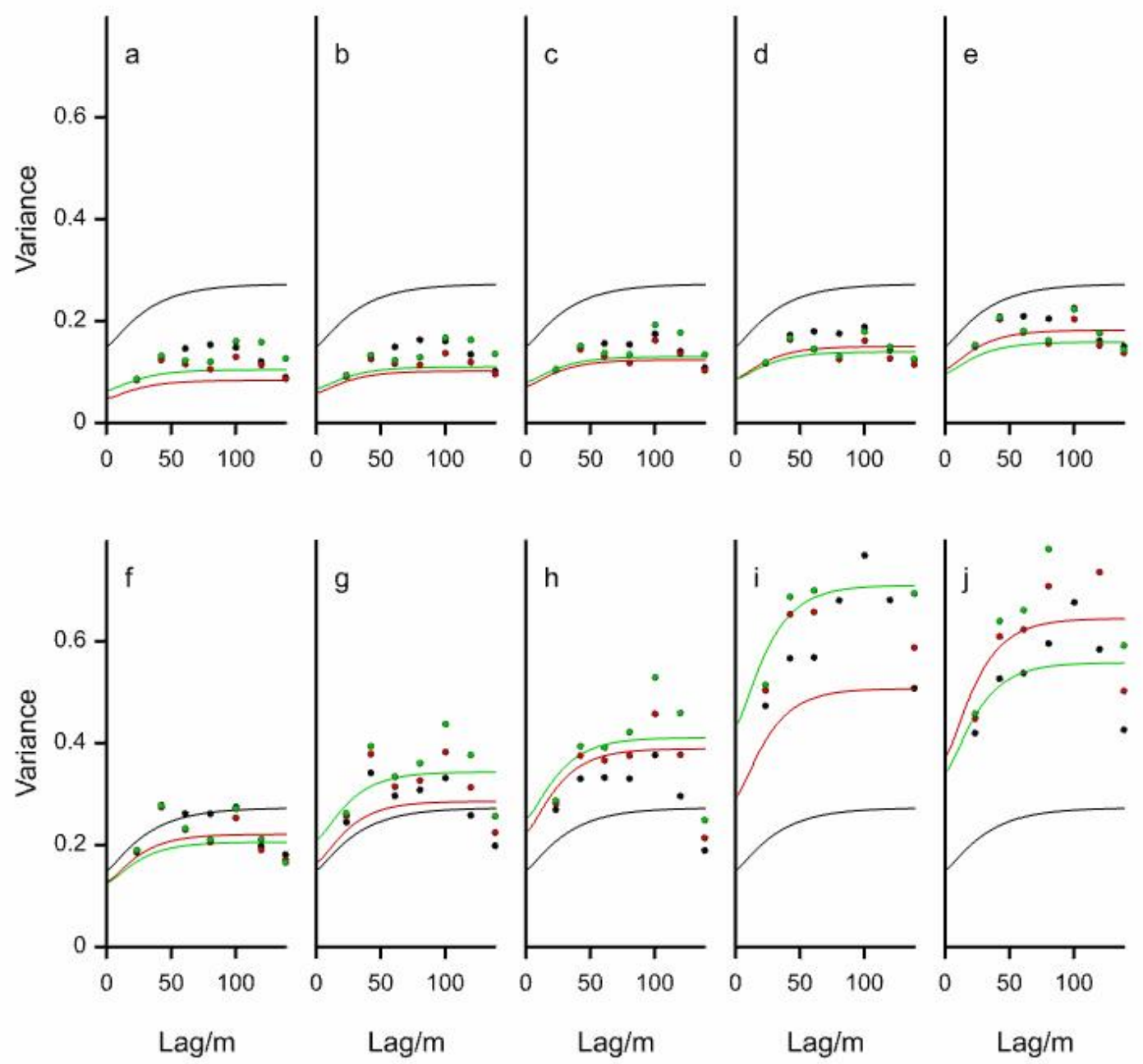

Fig. 4 (a)-(j) Horizontal method of moments point estimates (discs) and ML estimated product sum models (curves) for depths (a) 0.05, (b) 0.15, (c) 0.25, (d) 0.35, (e) 0.45, (f) 0.55, (g) 0.68, (h) 0.83, (i) 0.95 and (j) $1.05 \mathrm{~m}$. Scaling functions are constant (black), cubic (red) and discontinuous (green). 
Fig. 5 MSDR (mean square deviation ratio) against depth for the ML estimated product sum models with constant (black), cubic (red) and discontinuous (green) scaling functions. 


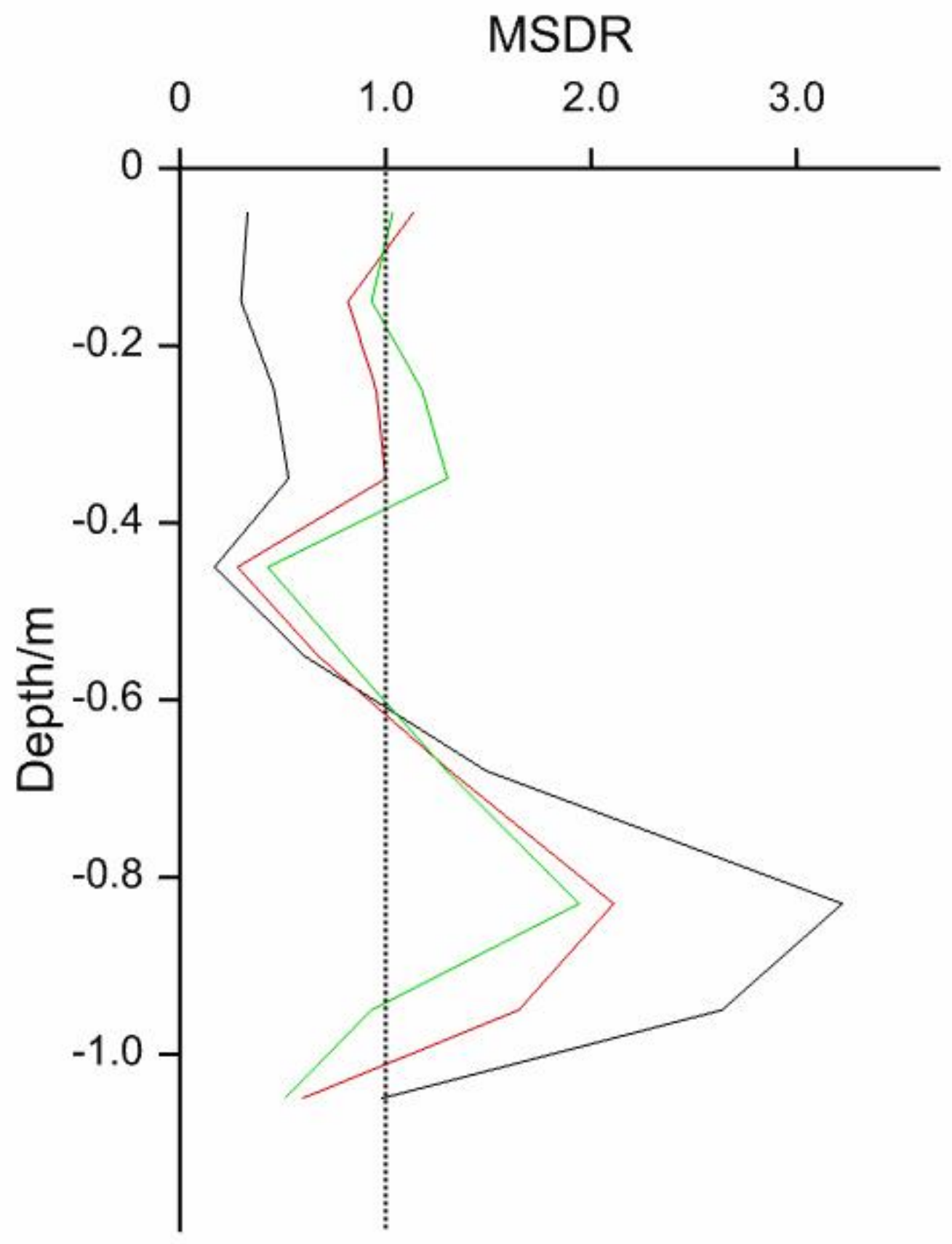



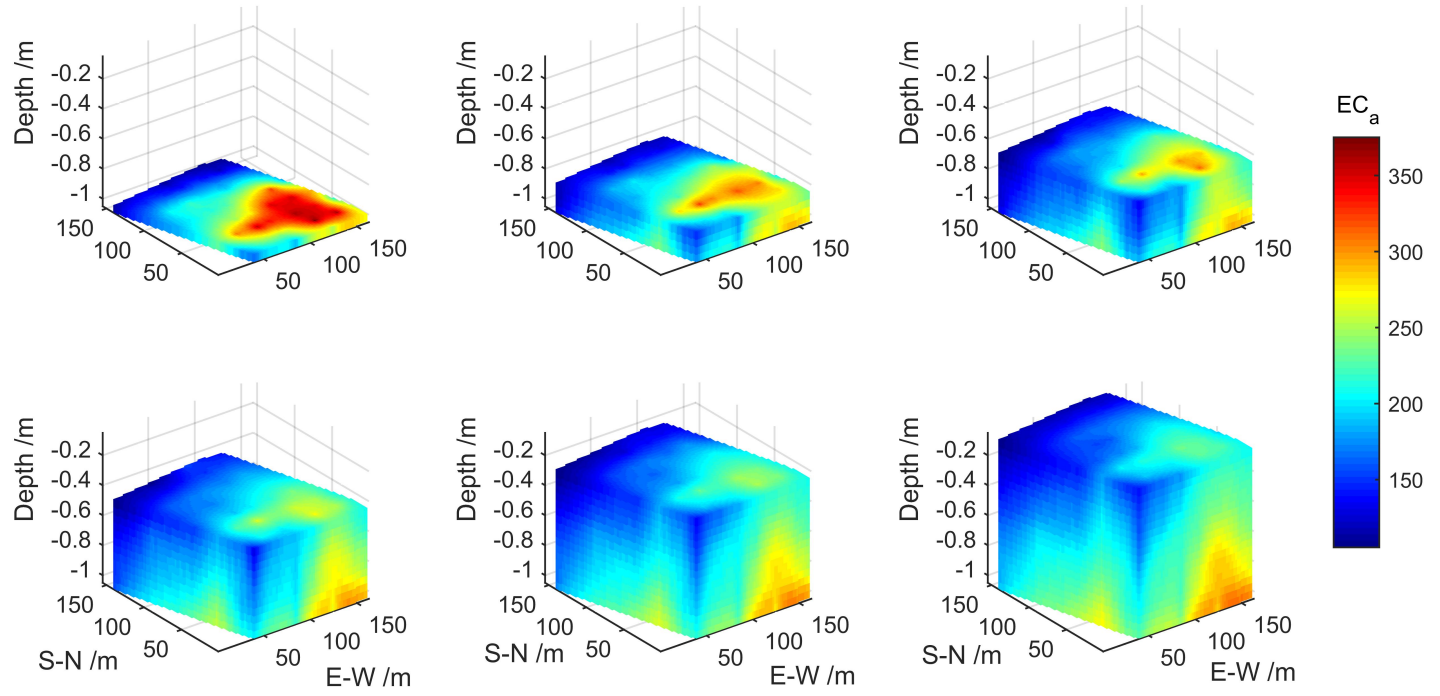

Fig. 6 Three-dimensional kriged predictions of $\mathrm{EC}_{\mathrm{a}}$ in $\mathrm{mS} \mathrm{m}^{-1}$. 

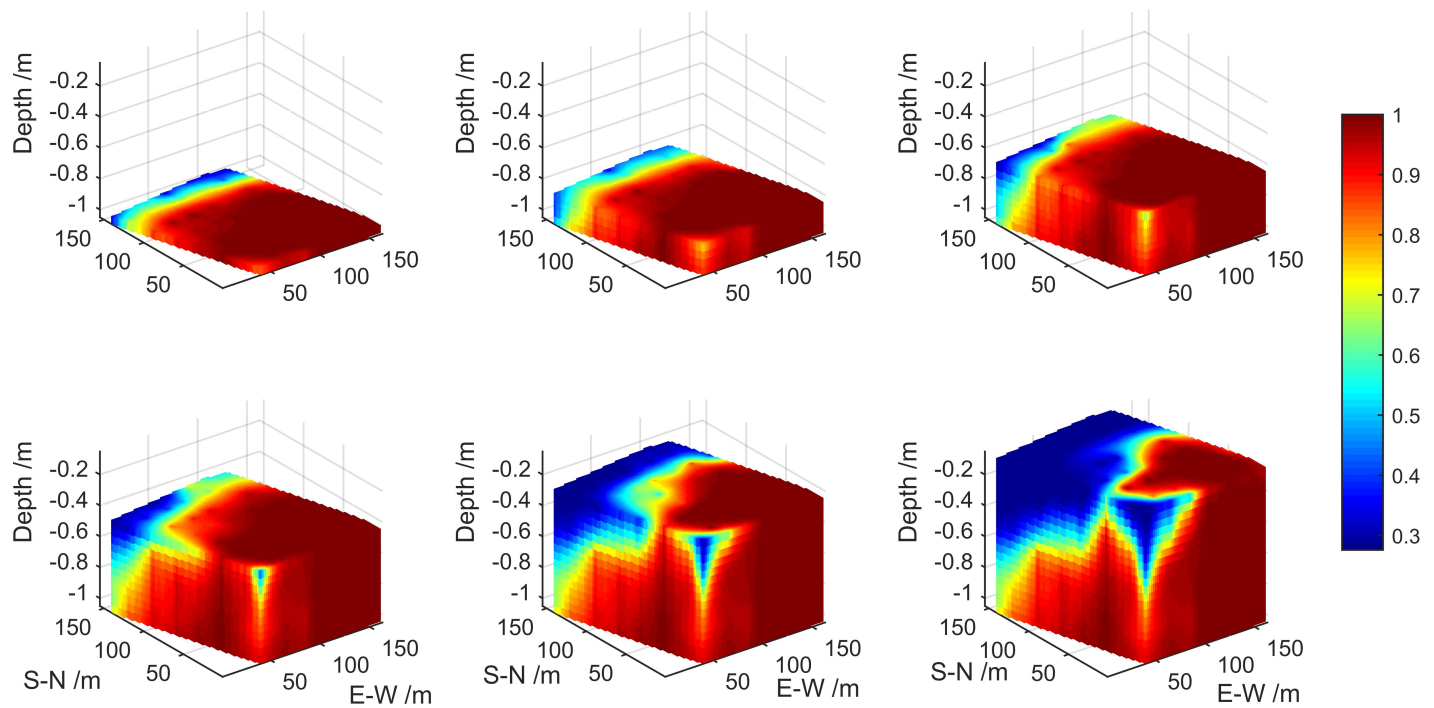

Fig. 7 The probability that $\mathrm{EC}_{\mathrm{a}}$ exceeds $123 \mathrm{mS} \mathrm{m}{ }^{-1}$. 